\title{
On hyperovals of polar spaces
}

\author{
Bart De Bruyn* \\ Ghent University, Department of Pure Mathematics and Computer Algebra, \\ Krijgslaan 281 (S22), B-9000 Gent, Belgium, E-mail: bdb@cage.ugent.be
}

\begin{abstract}
We derive lower and upper bounds for the size of a hyperoval of a finite polar space of rank 3 . We give a computer-free proof for the uniqueness, up to isomorphism, of the hyperoval of size 126 of $H(5,4)$ and prove that the near hexagon $\mathbb{E}_{3}$ has up to isomorphism a unique full embedding into the dual polar space $D H(5,4)$.
\end{abstract}

Dedicated to the memory of András Gács (1969-2009)

Keywords: hyperovals of polar spaces, (locally subquadrangular) hyperplanes, near hexagons, full embeddings

MSC2000: 51A50

\section{The main results}

Let $s, t \geq 1$. A generalized quadrangle $\mathcal{Q}$ of order $(s, t)$, or shortly a $\operatorname{GQ}(s, t)$, is a point-line geometry which satisfies the following properties: (i) every two distinct points are incident with at most one line; (ii) every line is incident with precisely $s+1$ points; (iii) every point is incident with precisely $t+1$ lines; (iv) for every non-incident point-line pair $(x, L)$, there exists a unique point on $L$ collinear with $x$. The generalized quadrangles belong to a larger class of geometrical structures, the so-called polar spaces, see Tits [28, Chapter 7].

Now, let $\mathcal{P}$ be one of the following polar spaces of rank $r \geq 2$ :

\footnotetext{
${ }^{*}$ The author is a Postdoctoral Fellow of the Research Foundation - Flanders (Belgium)
} 
(1) a generalized quadrangle of order $(s, t), s, t \geq 1(r=2)$;

(2) the polar space $W(2 r-1, q)$ of the subspaces of $\mathrm{PG}(2 r-1, q)$ which are totally isotropic with respect to a given symplectic polarity of $\mathrm{PG}(2 r-1, q)$;

(3) the polar space $Q(2 r, q)$ of the subspaces of $\mathrm{PG}(2 r, q)$ which lie on a given nonsingular parabolic quadric of $\mathrm{PG}(2 r, q)$;

(4) the polar space $Q^{+}(2 r-1, q)$ of the subspaces of PG $(2 r-1, q)$ which lie on a given nonsingular hyperbolic quadric of $\mathrm{PG}(2 r-1, q)$;

(5) the polar space $Q^{-}(2 r+1, q)$ of the subspaces of $\mathrm{PG}(2 r+1, q)$ which lie on a given nonsingular elliptic quadric of $\mathrm{PG}(2 r+1, q)$;

(6) the polar space $H\left(2 r-1, q^{2}\right)$ of the subspaces of $\mathrm{PG}\left(2 r-1, q^{2}\right)$ which lie on a given nonsingular Hermitian variety of $\mathrm{PG}\left(2 r-1, q^{2}\right)$;

(7) the polar space $H\left(2 r, q^{2}\right)$ of the subspaces of $\mathrm{PG}\left(2 r, q^{2}\right)$ which lie on a given nonsingular Hermitian variety of $\mathrm{PG}\left(2 r, q^{2}\right)$.

The polar spaces $W(2 r-1, q)$ and $Q(2 r, q)$ are isomorphic if and only if $q$ is even. A hyperoval of $\mathcal{P}$ is a non-empty set of points of $\mathcal{P}$ which intersects every singular line of $\mathcal{P}$ in either 0 or 2 points. Hyperovals of polar spaces arise in the context of locally polar spaces, see Buekenhout and Hubaut [6] and Pasechnik [21, 22, 23].

The first goal of this paper is to derive lower and upper bounds for the size of a hyperoval of the polar space $\mathcal{P}$ when its rank $r$ is equal to 3 . The solution for this problem in the case $r \neq 3$ is essentially known, see Propositions 2.1 and 2.2 of Section 2 .

Suppose $\mathcal{P}$ is one of the polar spaces $W(5, q), Q(6, q), Q^{+}(5, q), Q^{-}(7, q)$, $H\left(5, q^{2}\right), H\left(6, q^{2}\right)$. If $X$ is a hyperoval of $\mathcal{P}$ and $\alpha$ a singular plane meeting $X$, then $\alpha \cap X$ is a hyperoval of $\alpha$, i.e. a non-empty set of points of $\alpha$ meeting each line of $\alpha$ in either 0 or 2 points. The existence of hyperovals in $\alpha$ forces $q$ to be even, see e.g. Hirschfeld [16, Theorem 8.5].

Theorem 1.1 Suppose $q$ is even.

(1) If $X$ is a hyperoval of $Q(6, q) \cong W(5, q)$, then $q+2$ is a divisor of $|X|$ and $q^{4}+2 q^{2}+q+2 \leq|X| \leq q^{4}+2 q^{3}+q+2$. If the lower bound is achieved, then $q=2$ and $X$ is the complement of a hyperbolic quadric $Q^{+}(5,2)$ on $Q(6,2) \cong W(5,2)$.

(2) If $X$ is a hyperoval of $Q^{+}(5, q)$, then $q+2$ is a divisor of $|X|$ and $\frac{(q+2)\left(q^{2}+q+2\right)}{2} \leq|X| \leq\left(q^{2}+1\right)(q+2)$.

(3) If $X$ is a hyperoval of $Q^{-}(7, q)$, then $q+2$ is a divisor of $|X|$ and $q^{5}+q^{4}+q^{2}+q+2 \leq|X| \leq q^{5}+2 q^{4}+q+2$. If the lower bound is 
achieved, then $q=2$ and $X$ is the complement of a parabolic quadric $Q(6,2)$ on $Q^{-}(7,2)$.

(4) If $X$ is a hyperoval of $H\left(5, q^{2}\right)$, then $q^{2}+2$ is a divisor of $|X|$ and $q^{7}-q^{6}+q^{5}+q^{4}+q^{3}+q^{2}+2 \leq|X| \leq q^{7}+2 q^{5}+q^{2}+2$. If the lower bound is achieved, then $q=2$ and $X$ is a hyperoval of size 126 of $H(5,4)$.

(5) If $X$ is a hyperoval of $H\left(6, q^{2}\right)$, then $q^{2}+2$ is a divisor of $|X|$ and $q^{9}+q^{7}-q^{6}+q^{5}+q^{4}+q^{2}+2<|X| \leq q^{9}+2 q^{7}+q^{2}+2$.

If $\mathcal{P}$ is one of the polar spaces $Q(6,2), Q^{+}(5,2), Q^{-}(7,2)$, and $X$ is a hyperoval of $\mathcal{P}$, then the complement $\bar{X}$ of $X$ is a proper set of points of $\mathcal{P}$ which intersects each singular line in either 1 or 3 points, i.e. $\bar{X}$ is a hyperplane of $\mathcal{P}$. By Cohen and Shult [9, Theorem 5.12], every hyperplane of $\mathcal{P}$ is obtained by intersecting the corresponding quadric with a hyperplane of the ambient projective space.

All hyperovals of the polar spaces $Q(6,4), Q^{+}(5,4), Q^{-}(7,4), H(5,4)$ and $H(6,4)$ were classified by Pasechnik [21, Proposition 3.1] with the aid of a computer. In the special case of the polar space $H(5,4)$, he used a computer backtrack search to prove that there are, up to isomorphism, two classes of hyperovals in $H(5,4)$, a class of hyperovals consisting of 126 points and a class of hyperovals consisting of 162 points. Notice that the hyperovals of size 126 also arise in Theorem 1.1(4). In the present paper, we shall give a computer-free proof for the uniqueness, up to isomorphism, of the hyperoval of size 126 of $H(5,4)$.

Theorem 1.2 There exists up to isomorphism a unique hyperoval of size 126 of $H(5,4)$.

Pasini and Shpectorov [24] proved that there exists a connection between the hyperovals of size 126 of $H(5,4)$ and the so-called locally subquadrangular hyperplanes of the dual polar space $D H(5,4)$ associated with $H(5,4)$. We will use this connection to give a computer-free proof of Theorem 1.2. During this proof, we will also make use of the uniqueness (up to isomorphism) of the full embedding of the near hexagon $\mathbb{E}_{3}$ (to be defined later) in the dual polar space $D H(5,4)$. By a full embedding of a point-line geometry $\mathcal{S}_{1}$ into a point-line geometry $\mathcal{S}_{2}$, we mean an injective mapping $e$ from the point-set of $\mathcal{S}_{1}$ to the point-set of $\mathcal{S}_{2}$ mapping lines of $\mathcal{S}_{1}$ to full lines of $\mathcal{S}_{2}$. 
Theorem 1.3 Let $e_{1}$ and $e_{2}$ be two full embeddings of $\mathbb{E}_{3}$ into the dual polar space $D H(5,4)$. Then there exists a unique automorphism $\theta$ of $D H(5,4)$ such that $\theta \circ e_{1}=e_{2}$.

Remark. Suppose $X$ is a hyperoval of size 126 of $H(5,4)$. Let $\Gamma_{X}$ be the graph whose vertices are the points of $X$, two points of $X$ being adjacent whenever they are collinear on $H(5,4)$. It is easily proved that the graph $\Gamma_{X}$ satisfies the following properties: (i) every maximal clique has six points; (ii) for every maximal clique $C$ and every vertex $p \notin C$, there are exactly two neighbours of $p$ in $C$; (iii) no vertex is adjacent to all other vertices. These conditions imply that $\Gamma_{X}$ is a so-called Zara graph with clique size 6 and nexus 2 (Zara [29]). Blokhuis and Brouwer [3] proved without a computer that there exists, up to isomorphism, a unique graph satisfying the conditions (i), (ii) and (iii) above. By this result, we know that the graph $\Gamma_{X}$ is uniquely determined (up to an isomorphism of graphs). Theorem 1.2 says that also the hyperoval $X$ is uniquely determined (up to an isomorphism of the polar space $H(5,4))$. Another computer free characterization of the above Zara graph was obtained in Pasechnik [20, Proposition 2.6]: the Zara graph is, up to isomorphism, the unique graph $\Gamma$ which satisfies the following conditions: (a) $\Gamma$ is isomorphic to a subgraph of the collinearity graph of $H(5,4)$; (b) $\Gamma$ is locally $H(3,4)$; (c) for any pair $(x, y)$ of nonadjacent vertices of $\Gamma$, the graph induced on the common neighbours of $x$ and $y$ is isomorphic to $K_{3,3} \cup K_{3,3} \cup K_{3,3}$ (the disjoint union of three bipartite graphs $K_{3,3}$ ).

\section{Hyperovals in polar spaces of rank $r \neq 3$}

Suppose $\mathcal{Q}$ is a generalized quadrangle of order $(s, t)$. If $X$ is a set of points of $\mathcal{Q}$, then $X^{\perp}$ denotes the set of all points of $\mathcal{Q}$ which are collinear with every point of $X$. We will denote $\left(X^{\perp}\right)^{\perp}$ also by $X^{\perp \perp}$. If $x$ and $y$ are noncollinear points of $\mathcal{Q}$, then $\left|\{x, y\}^{\perp \perp}\right| \leq t+1$. If equality occurs, then the pair $\{x, y\}$ is called regular. If this is the case, then every line of $\mathcal{Q}$ intersects $\{x, y\}^{\perp} \cup\{x, y\}^{\perp \perp}$ in either 0 or 2 points. If a $\operatorname{GQ}(s, t)$ has a regular pair of non-collinear points, then by Payne and Thas [25, 1.3.6(i)] either $s=1$ or $s \geq t$. An ovoid of a $\mathrm{GQ}(s, t)$ is a set of points meeting each line in a unique point. Such an ovoid contains precisely $s t+1$ points. For more background information on generalized quadrangles, we refer to the standard work [25]. 
Lower and upper bounds for the size of a generalized quadrangle of order $(s, t)$ were obtained in Cameron, Hughes, Pasini [8, Lemmas 3.9 and 3.11] and Del Fra, Ghinelli, Payne [14, Theorems 2.1 and 2.2].

Proposition $2.1([\mathbf{8}, \mathbf{1 4}])$ Let $\mathcal{Q}$ be a generalized quadrangle of order $(s, t)$ and let $X$ be a hyperoval of $\mathcal{Q}$. Then

(1) 2 is a divisor of $|X|$;

(2) We have $|X| \geq 2(t+1)$, with equality if and only if there exists a regular pair $\{x, y\}$ of non-collinear points of $\mathcal{Q}$ such that $X=\{x, y\}^{\perp} \cup$ $\{x, y\}^{\perp \perp}$.

(3) We have $|X| \geq(t-s+2)(s+1)$. If equality holds then every point outside $X$ is incident with precisely $\frac{t-s}{2}+1$ lines which meet $X$ (hence $s \equiv t$ $(\bmod 2))$.

(4) We have $|X| \leq 2($ st +1$)$, with equality if and only if every line of $\mathcal{Q}$ intersects $X$ in precisely 2 points.

Remark. If $s=1$, then the lower bounds and upper bound for $|X|$ mentioned in Proposition 2.1 coincide with $2(t+1)$. Indeed, a GQ $(1, t)$ has only one hyperoval: the whole point-set. If $s=t$, then the two lower bounds for $|X|$ mentioned in Proposition 2.1 coincide. If $t>s \neq 1$, then the lower bound for $|X|$ mentioned in Proposition 2.1(3) is better than the lower bound mentioned in Proposition 2.1(2). The opposite occurs if $t<s$.

Examples. (I) Let $\mathcal{Q}$ be a generalized quadrangle of order $(2, t)$. Then the hyperovals of $\mathcal{Q}$ are precisely the complements of the hyperplanes of $\mathcal{Q}$. [By a hyperplane of a point-line geometry we mean a proper subset of the point-set which meets every line in either a point or the whole line.] If $t=1$ (so, $\mathcal{Q}$ is the $3 \times 3$-grid), then every hyperoval $X$ of $\mathcal{Q}$ is either an ordinary 4-gon or the complement of an ovoid; hence, $|X| \in\{4,6\}$. If $t=2$ (so, $\mathcal{Q} \cong W(2):=W(3,2))$, then every hyperoval is either the complement of a $(3 \times 3)$-grid, the complement of an ovoid, or the set of points at distance 2 from a given point; hence $|X| \in\{6,8,10\}$. If $t=4$ (so, $\mathcal{Q} \cong Q^{-}(5,2)$ ), then every hyperoval is either the complement of a $W(2)$-subquadrangle or the set of points at distance 2 from a given point; hence $|X| \in\{12,16\}$. Except for the missing values " 14 " and " 18 " in the case of $Q^{-}(5,2)$, the above spectra of hyperoval sizes are precisely the spectra as predicted by Proposition 2.1.

(II) Makhnev [18] classified all hyperovals of the generalized quadrangle $H(3,4)$ (which has order $(4,2))$ and found that for every $\mu \in\{6,8,10,12,14$, 
$16,18\}$ there exists a hyperoval of size $\mu$. Hence, the spectrum of hyperoval sizes is precisely as predicted by Proposition 2.1 .

(III) Makhnev [19] classified hyperovals of the unique generalized quadrangle of order $(3,5)$ and found that for every $\mu \in\{16,18,20,22,24,30,32\}$, there exists a hyperoval of size $\mu$. Notice that by Proposition 2.1 we should have $2 \mid \mu$ and $16 \leq \mu \leq 32$.

(IV) Pasechnik [22] classified with the aid of a computer all hyperovals of the generalized quadrangles $W(3):=W(3,3)$ and $Q(4,3)$ (which both have order $(3,3))$. He found that: (i) for every $\mu_{1} \in\{8,12,16,20\}$, there exists, up to isomorphism, a unique hyperoval of size $\mu_{1}$ of $W(3)$; (ii) for every $\mu_{2} \in\{10,12,14,16,18,20\}$, there exists, up to isomorphism, a unique hyperoval of size $\mu_{2}$ of $Q(4,3)$. Notice that by Proposition 2.1 the size $\mu$ of a hyperoval of a generalized quadrangle of order $(3,3)$ must satisfy $2 \mid \mu$ and $8 \leq \mu \leq 20$.

(V) Pasechnik [23] classified with the aid of a computer all hyperovals of the generalized quadrangle $Q^{-}(5,3)$ (which has order $(3,9)$ ) and found that for every $\mu \in\{32,40,56\}$ there exists a hyperoval of size $\mu$. Notice that by Proposition 2.1 we should have $2 \mid \mu$ and $32 \leq \mu \leq 56$.

If the rank of the polar space is at least 4, then a classification of the hyperovals is readily obtained. Such a classification would follow from Pasechnik [21, Lemma 2.2], but a more direct approach can also be given.

Proposition 2.2 (1) Let $\mathcal{P}$ be one of the polar spaces $W(2 r-1, q)$ ( $q$ odd), $H\left(2 r-1, q^{2}\right), H\left(2 r, q^{2}\right)$, where $r \geq 4$. Then $\mathcal{P}$ has no hyperovals.

(2) Let $\mathcal{P}$ be one of the following polar spaces of rank $r \geq 4$ : (a) $Q(2 r, q)$; (b) $Q^{+}(2 r-1, q)$; (c) $Q^{-}(2 r+1, q)$. Let $\Pi$ be the ambient projective space of $\mathcal{P}$. If $X$ is a hyperoval of $\mathcal{P}$, then $q=2$ and there exists a hyperplane $\pi$ of $\Pi$ such that $X$ consists of those points of $\mathcal{P}$ not contained in $\pi$.

Proof. Let $\mathcal{P}$ be one of the polar spaces mentioned in (1) or (2), and let $X$ be a hyperoval of $\mathcal{P}$. If $\alpha$ is a maximal singular subspace of $\mathcal{P}$ meeting $X$, then $\alpha \cap X$ is a hyperoval of $\alpha$, i.e. a non-empty set of points of $\alpha$ meeting each line in either 0 or 2 points. Notice that $\alpha \cong \operatorname{PG}\left(r-1, q^{\prime}\right)$ where $q^{\prime} \in\left\{q, q^{2}\right\}$. Now, a hyperoval of $\mathrm{PG}\left(r-1, q^{\prime}\right), r-1 \geq 3$, can only exists if $q^{\prime}=2$ (in which case it is the complement of a hyperplane), see e.g. Hirschfeld [15, Lemma 16.1.4]. Hence, $\mathcal{P}$ is one of the following: (a) $Q(2 r, 2) ;(b) Q^{+}(2 r-1,2) ;(c)$ $Q^{-}(2 r+1,2)$. Now, the complement of $X$ is a hyperplane of $\mathcal{P}$. By Cohen 
and Shult [9, Theorem 5.12], a hyperplane of $\mathcal{P}$ arises by intersecting the point-set of $\mathcal{P}$ with a hyperplane of $\Pi$.

\section{Proof of Theorem 1.1}

Let $\mathcal{P}$ be one of the following polar spaces of rank 3 (with $q$ even): (i) $W(5, q) \cong Q(6, q)$; (ii) $Q^{+}(5, q)$; (iii) $Q^{-}(7, q)$; (iv) $H(5, q)$ ( $q$ square); (v) $H(6, q)$ ( $q$ square). Then every singular plane of $\mathcal{P}$ is isomorphic to $\mathrm{PG}(2, q)$. Let $s+1$ denote the constant number of singular planes through a given singular line of $\mathcal{P}$. Then $s=q$ if $\mathcal{P}=W(5, q), s=1$ if $\mathcal{P}=Q^{+}(5, q), s=q^{2}$ if $\mathcal{P}=Q^{-}(7, q), s=\sqrt{q}$ if $\mathcal{P}=H(5, q)$ and $s=q^{\frac{3}{2}}$ if $\mathcal{P}=H(6, q)$. Now, $\mathcal{P}$ has $\left(s q^{2}+1\right)\left(q^{2}+q+1\right)$ points, $(s q+1)\left(s q^{2}+1\right)\left(q^{2}+q+1\right)$ singular lines and $(s+1)(s q+1)\left(s q^{2}+1\right)$ singular planes. Every point of $\mathcal{P}$ is contained in $(q+1)(s q+1)$ singular lines and $(s+1)(s q+1)$ singular planes.

Lemma 3.1 Let $X$ be a hyperoval of $\mathcal{P}$. Then $|X| \geq \frac{(q+2)\left(s q^{2}+s q+2\right)}{2}$.

Proof. Let $\alpha$ be a singular plane of $\mathcal{P}$ containing a point of $X$. Then $|\alpha \cap X|=q+2$. There are $\frac{(q+2)(q+1)}{2}$ lines in $\alpha$ which intersect $X$ in two points, each such line is contained in $s$ singular planes distinct from $\alpha$ and each such singular plane contains $q$ points of $X \backslash \alpha$. The $\frac{(q+2)(q+1)}{2} s q$ points which arise in this way are mutually distinct. Hence, $|X| \geq(q+2)+\frac{(q+2)(q+1)}{2} \cdot s q=$ $\frac{(q+2)\left(s q^{2}+s q+2\right)}{2}$.

Lemma 3.2 If $X$ is a hyperoval of $\mathcal{P}$ and $\alpha$ is a singular plane such that $|\alpha \cap X|=q+2$, then there are precisely $\frac{|X|}{q+2}-\frac{s q^{2}+s q+2}{2}$ singular planes which intersect $X$ in $q+2$ points and $\alpha$ in a line disjoint from $X$. Hence, $q+2$ is a divisor of $|X|$.

Proof. For every point $x$ of $\mathcal{P}$ outside $\alpha$, let $\alpha_{x}$ denote the unique singular plane through $x$ meeting $\alpha$ in a line. There are $|X|-\frac{(q+2)\left(s q^{2}+s q+2\right)}{2}$ points $x \in X \backslash \alpha$ such that $\alpha_{x} \cap \alpha$ is disjoint from $\alpha \cap X$. Each such point $x \in X \backslash \alpha$ gives rise to a suitable plane $\alpha_{x}$. Each such plane is counted $q+2$ times.

Lemma 3.3 Let $X$ be a hyperoval of $\mathcal{P}$. Then

$$
(s q-q+2)\left(q^{2}+q+1\right) \leq|X| \leq\left(s q^{2}+1\right)(q+2) .
$$


If $(s q-q+2)\left(q^{2}+q+1\right)=|X|$, then every singular line of $\mathcal{P}$ disjoint from $X$ is contained in precisely $\frac{s q-q+2}{q+2}$ singular planes which meet $X$ in $q+2$ points. If $|X|=\left(s q^{2}+1\right)(q+2)$, then every singular plane of $\mathcal{P}$ intersects $X$ in precisely $q+2$ points.

Proof. Let $\mathcal{L}$ denote the set of singular lines of $\mathcal{P}$ disjoint from $X$. For every $L \in \mathcal{L}$, let $n_{L}$ denote the number of singular planes through $L$ intersecting $X$ in precisely $q+2$ points. The total number of elements of $\mathcal{L}$ is equal to

$$
\sum_{L \in \mathcal{L}} 1=(s q+1)\left(s q^{2}+1\right)\left(q^{2}+q+1\right)-\frac{|X| \cdot(q+1)(s q+1)}{2} .
$$

Counting in two different ways the number of pairs $(L, \alpha)$, where $L \in \mathcal{L}$ and $\alpha$ a singular plane through $L$ intersecting $X$ in $q+2$ points, gives

$$
\sum_{L \in \mathcal{L}} n_{L}=\frac{|X| \cdot(s+1)(s q+1)}{q+2} \cdot \frac{q^{2}-q}{2} .
$$

Counting in two different ways the number of triples $\left(L, \alpha_{1}, \alpha_{2}\right)$ where $L \in \mathcal{L}$ and $\alpha_{1}$ and $\alpha_{2}$ two singular planes such that $\left|\alpha_{1} \cap X\right|=\left|\alpha_{2} \cap X\right|=q+2$ and $L=\alpha_{1} \cap \alpha_{2}$, gives (recall Lemma 3.2)

$$
\sum_{L \in \mathcal{L}} n_{L}\left(n_{L}-1\right)=\frac{|X| \cdot(s+1)(s q+1)}{q+2}\left(\frac{|X|}{q+2}-\frac{s q^{2}+s q+2}{2}\right) .
$$

By (2) and (3), we find $\sum_{L \in \mathcal{L}} n_{L}^{2}=\frac{|X| \cdot(s+1)(s q+1)}{q+2}\left(\frac{|X|}{q+2}-\frac{(s q-q+2)(q+1)}{2}\right)$. By the Cauchy-Schwartz inequality $\left(\sum 1\right) \cdot\left(\sum n_{L}^{2}\right) \geq\left(\sum n_{L}\right)^{2}$, we find $(|X|-$ $\left.(s q-q+2)\left(q^{2}+q+1\right)\right) \cdot\left(|X|-\left(s q^{2}+1\right)(q+2)\right) \leq 0$. Since $\left(s q^{2}+1\right)(q+2)-$ $(s q-q+2)\left(q^{2}+q+1\right)=q(q-1)(q+s)>0$, we find $(s q-q+2)\left(q^{2}+q+1\right) \leq$ $|X| \leq\left(s q^{2}+1\right)(q+2)$. If equality holds in either the lower or the upper bound, then $n_{L}$ is independent from the chosen line $L \in \mathcal{L}$.

If $|X|=(s q-q+2)\left(q^{2}+q+1\right)$, then $\sum_{L \in \mathcal{L}} 1=\frac{(s+1) q(q-1)(s q+1)\left(q^{2}+q+1\right)}{2}$ and $\sum_{L \in \mathcal{L}} n_{L}=\frac{(s q-q+2)\left(q^{2}+q+1\right)(s+1)(s q+1)\left(q^{2}-q\right)}{2(q+2)}$. Hence, every singular line disjoint from $X$ is contained in $\frac{\sum n_{L}}{\sum 1}=\frac{s q-q+2}{q+2}$ singular planes which intersect $X$ in precisely $q+2$ points.

In a similar way one proves that if $|X|=\left(s q^{2}+1\right)(q+2)$, then every singular line disjoint from $X$ is contained in precisely $s+1$ singular planes 
which intersect $X$ in $q+2$ points. Hence, every singular plane of $\mathcal{P}$ intersects $X$ in $q+2$ points.

We will now give a proof of Theorem 1.1.

First, suppose that $\mathcal{P}=W(5, q) \cong Q(6, q)$. Then $q^{4}+2 q^{2}+q+2 \leq|X| \leq$ $q^{4}+2 q^{3}+q+2$ by Lemma 3.3. If $|X|=q^{4}+2 q^{2}+q+2$, then also by Lemma $3.3, q+2$ is a divisor of $s q-q+2=q^{2}-q+2=(q+2)(q-3)+8$. Hence, $q=2, \mathcal{P}=Q(6,2)$ and $|X|=28$. Now, $X$ is the complement of a hyperplane of $\mathcal{P}$ and since $|X|=28$, this hyperplane is necessarily a hyperbolic quadric $Q^{+}(5,2)$ on $Q(6,2)$ (recall Cohen and Shult [9]).

If $\mathcal{P}=Q^{+}(5, q)$, then by Lemmas 3.1 and $3.3, \frac{(q+2)\left(q^{2}+q+2\right)}{2} \leq|X| \leq$ $\left(q^{2}+1\right)(q+2)$. (Notice that the lower bound of Lemma 3.3 is weaker in this case.)

If $\mathcal{P}=Q^{-}(7, q)$, then $q^{5}+q^{4}+q^{2}+q+2 \leq|X| \leq q^{5}+2 q^{4}+q+2$ by Lemma 3.3. If $|X|=q^{5}+q^{4}+q^{2}+q+2$, then also by Lemma $3.3, q+2$ is a divisor of $s q-q+2=q^{3}-q+2=(q+2)\left(q^{2}-2 q+3\right)-4$. Hence, $q=2$, $\mathcal{P}=Q^{-}(7,2)$ and $|X|=56$. Now, $X$ is the complement of a hyperplane of $\mathcal{P}$ and since $|X|=56$, this hyperplane is necessarily a parabolic quadric $Q(6,2)$ on $Q^{-}(7,2)$.

Suppose $\mathcal{P}=H\left(5, q^{2}\right)$. (For reasons of convenience, we have replaced $q$ by $q^{2}$.) By Lemma 3.3, $q^{7}-q^{6}+q^{5}+q^{4}+q^{3}+q^{2}+2 \leq|X| \leq q^{7}+2 q^{5}+q^{2}+2$. If the lower bound is achieved then by Lemma $3.3, q^{2}+2$ is a divisor of $s q^{2}-q^{2}+2=q^{3}-q^{2}+2=\left(q^{2}+2\right)(q-1)-(2 q-4)$. Hence, $q=2$ and $|X|=126$.

Finally, suppose that $\mathcal{P}=H\left(6, q^{2}\right)$. By Lemma $3.3, q^{9}+q^{7}-q^{6}+q^{5}+$ $q^{4}+q^{2}+2 \leq|X| \leq q^{9}+2 q^{7}+q^{2}+2$. Suppose the lower bound occurs. Then by Lemma $3.3, q^{2}+2$ is a divisor of $s q^{2}-q^{2}+2=q^{5}-q^{2}+2=$ $\left(q^{3}-2 q-1\right)\left(q^{2}+2\right)+4 q+4$. Hence, $q=2$. But this is impossible, since the polar space $H(6,4)$ does not admit hyperovals by Pasechnik [21, Proposition 3.1].

\section{Proof of Theorem 1.3}

\subsection{Near polygons and hyperplanes of dual polar spaces}

A point-line geometry $\mathcal{S}$ is called a near polygon if for every point $x$ and every line $L$, there exists a unique point on $L$ nearest to $x$. Here, distances 
$\mathrm{d}(\cdot, \cdot)$ are measured in the collinearity graph $\Gamma$ of $\mathcal{S}$. If $d$ is the diameter of $\Gamma$, then the near polygon is called a near $2 d$-gon. A near 0 -gon consists of a unique point (no lines) and a near 2-gon is a line. The near quadrangles are precisely the generalized quadrangles.

If $x$ is a point of $\mathcal{S}$ and $i \in \mathbb{N}$, then $\Gamma_{i}(x)$ denotes the set of points of $\mathcal{S}$ at distance $i$ from $x$. For every point $x$ of $\mathcal{S}$, we define $x^{\perp}:=\{x\} \cup \Gamma_{1}(x)$. A near polygon is called dense if every line is incident with at least three points and if every two points at distance 2 have at least two common neighbours.

Let $\mathcal{S}$ be a near polygon and let $X$ be a set of points of $\mathcal{S}$. $X$ is called a subspace if it contains all the points of a line as soon as it contains at least two points of that line. If $X$ is a subspace of $\mathcal{S}$, then the lines of $\mathcal{S}$ which have all their points in $X$ define a subgeometry $\widetilde{X}$ of $\mathcal{S}$ on the point-set $X$. A set $X$ of points of $\mathcal{S}$ is called convex if it contains all the points on a shortest path between any two of its points. If $C$ is a convex subspace of $\mathcal{S}$, then $\widetilde{C}$ is also a near polygon. By Brouwer and Wilbrink [5, Theorem 4], every two points $x_{1}$ and $x_{2}$ of a dense near polygon are contained in a unique convex subspace $\left\langle x_{1}, x_{2}\right\rangle$ of diameter $\mathrm{d}\left(x_{1}, x_{2}\right)$. These convex subspaces are called quads if $\mathrm{d}\left(x_{1}, x_{2}\right)=2$. The existence of quads in dense near polygons was already proved in Shult and Yanushka [27, Proposition 2.5]. Every two distinct intersecting lines $L$ and $M$ of a dense near polygon are contained in a unique quad which we will denote by $\langle L, M\rangle$. If $x$ is a point of a dense near polygon $\mathcal{S}$, then the lines and quads of $\mathcal{S}$ through $x$ define a linear space $\operatorname{Res}(x)$ which is called the local space at $x$. For more background information on near polygons, we refer to the book [11].

By Brouwer et al. [4], there exists up to isomorphism a unique dense near hexagon which satisfies the following properties: (A) Every line is incident with precisely 3 points; (B) Every local space is isomorphic to $\overline{W(2)}$, the linear space obtained from the generalized quadrangle $W(2)$ by adding its ovoids as extra lines. This near hexagon, which we will denote by $\mathbb{E}_{3}$, was first constructed in [1]. An alternative and purely geometrical proof of the above classification result was also obtained in $[10$, Section $3(\mathrm{II})]$. As a consequence of the main result of [10], it was proved in that paper that there exists up to isomorphism at most one dense near hexagon which satisfies the properties (A) and (B) above. The uniqueness then follows from the existence. An explicit construction of the near hexagon $\mathbb{E}_{3}$ will be given below. The near hexagon $\mathbb{E}_{3}$ has 567 points. Every quad of $\mathbb{E}_{3}$ is isomorphic to either $W(2)$ or $Q^{-}(5,2)$. If $x$ is a point of $\mathbb{E}_{3}$ at distance 2 from a $W(2)$-quad $Q$, then 
$\Gamma_{2}(x) \cap Q$ is an ovoid of $\widetilde{Q}$. Moreover, for every $z \in \Gamma_{2}(x) \cap Q,\langle x, z\rangle$ is a $W(2)$-quad of $\mathbb{E}_{3}$.

Recall that a full embedding of a point-line geometry $\mathcal{S}_{1}$ into a pointline geometry $\mathcal{S}_{2}$ is an injective mapping $e$ from the point-set of $\mathcal{S}_{1}$ to the point-set of $\mathcal{S}_{2}$ mapping lines of $\mathcal{S}_{1}$ to full lines of $\mathcal{S}_{2}$. A full embedding is called isometric if it preserves the distances between points. By Huang [17, Corollary 3.3], a full embedding $e$ between two dense near $2 n$-gons is isometric if and only if there exist two opposite points $x_{1}$ and $x_{2}$ in $\mathcal{S}_{1}$ such that $e\left(x_{1}\right)$ and $e\left(x_{2}\right)$ are also opposite points of $\mathcal{S}_{2}$. (Two points are said to be opposite if they lie at maximal distance $n$ from each other.)

With every polar space $\mathcal{P}$ of rank $r \geq 2$, there is associated a near $2 r$ gon $\Delta$ which is called a dual polar space (Cameron [7]). The points of $\Delta$ are the maximal singular subspaces of $\mathcal{P}$, the lines are the next-to-maximal singular subspaces of $\mathcal{P}$, and incidence is reverse containment. Every convex subspace of $\Delta$ consists of the set of maximal (i.e. $(r-1)$-dimensional) singular subspaces of $\mathcal{P}$ which contain a given singular subspace of $\mathcal{P}$. If the dimension of this singular subspace of $\mathcal{P}$ is $r-3$, then the corresponding convex subspace of $\Delta$ is a quad. Recall that a hyperplane of $\Delta$ is a proper subset of the point-set of $\Delta$ which intersects each line in either a singleton or the whole line. By Blok and Brouwer [2, Theorem 7.3] or Shult [26, Lemma 6.1], every hyperplane of a thick dual polar space is a maximal subspace. If $H$ is a hyperplane of $\Delta$, then for every point $x$ of $H, \Lambda_{H}(x)$ denotes the set of lines through $x$ contained in $H$. We will regard $\Lambda_{H}(x)$ as a set of points of $\operatorname{Res}(x)$. A hyperplane $H$ of $\Delta$ is called locally subquadrangular if every quad $Q$ of $\Delta$ not contained in $H$ intersects $H$ in a (nondegenerate) subquadrangle of $\widetilde{Q}$.

We will denote a dual polar space by putting a " $\mathrm{D}$ " in front of the name of the corresponding polar space. E.g., $D Q(2 r, 2), r \geq 2$, denotes the dual polar space associated with $Q(2 r, 2)$. If $Q$ is a quad of $D Q(2 r, 2)$, then $\widetilde{Q} \cong W(2)$. If $x$ is a point of the dual polar space $D H(5,4)$, then $\operatorname{Res}(x) \cong \mathrm{PG}(2,4)$, $\left|\Gamma_{0}(x)\right|=1,\left|\Gamma_{1}(x)\right|=42,\left|\Gamma_{2}(x)\right|=336$ and $\left|\Gamma_{3}(x)\right|=512$. Every quad of $D H(5,4)$ is isomorphic to $Q^{-}(5,2)$. For every quad $Q$ of $D H(5,4)$ and for every point $x \notin Q$, there exists a unique point $x^{\prime} \in Q$ collinear with $x$ and $\mathrm{d}(x, y)=1+\mathrm{d}\left(x^{\prime}, y\right)$ for every point $y$ of $Q$.

Pasini and Shpectorov [24] used hyperovals of size 126 of $H(5,4)$ to construct locally subquadrangular hyperplanes of $D H(5,4)$. We take the freedom to give another construction yielding locally subquadrangular hyperplanes of $\operatorname{DH}(5,4)$. Consider in $\mathrm{PG}(6,2)$ a nonsingular parabolic quadric 
$Q(6,2)$, let $k$ denote the kernel of this quadric and let $\pi$ be a hyperplane of $\mathrm{PG}(6,2)$ intersecting $Q(6,2)$ in an elliptic quadric $Q^{-}(5,2)$. The projection from the kernel $k$ on the hyperplane $\pi$ defines an isomorphism between the polar space $Q(6,2)$ and the symplectic polar space $W(5,2)$ associated to a suitable symplectic polarity of $\pi$. Any set of points of $W(5,2)$ which is isomorphic to the subset $\pi \backslash Q^{-}(5,2)$ of $\pi$ is called an elliptic set of points of $W(5,2)$. A set of quads of $D W(5,2)$ corresponding to an elliptic set of points of $W(5,2)$ is called an elliptic set of quads of $D W(5,2)$.

It is well-known that the dual polar space $D W(5,2)$ can be isometrically embedded into the dual polar space $D H(5,4)$. In fact up to isomorphism there exists a unique such isometric embedding, see De Bruyn [12, Theorem 1.5]. Now, let $D W(5,2)$ be isometrically embedded into $D H(5,4)$ and for every quad $Q$ of $D W(5,2)$, let $\bar{Q}$ denote the unique quad of $D H(5,4)$ containing all points of $Q$. If $A$ is an elliptic set of quads of $D W(5,2)$, then by

De Bruyn [13, Theorem 1.5 + Proposition 2.17], $H:=D W(5,2) \cup\left(\bigcup_{Q \in A} \bar{Q}\right)$ is a hyperplane of $D H(5,4)$ with the property that $\left|\Lambda_{H}(x)\right|=15$ for every point $x \in H$. The hyperplane $H$ is easily seen to be locally subquadrangular: if $L$ is a line of $D H(5,4)$ having a unique point $x$ in common with $H$, then each of the 5 quads through $L$ contributes at most 3 (and hence precisely 3 ) to the total value of $\left|\Lambda_{H}(x)\right|=15$; so, every quad through $L$ intersects $H$ in a $W(2)$-subquadrangle. The point-line geometry $\widetilde{H}$ induced on $H$ is isomorphic to the near hexagon $\mathbb{E}_{3}$ (see e.g. Corollary 5.5 which we will prove in Section 5.1).

\subsection{Full embeddings of $\mathbb{E}_{3}$ into $D H(5,4)$}

Let $e$ be an arbitrary full embedding of the near hexagon $\mathbb{E}_{3}$ into the dual polar space $D H(5,4)$. [E.g., the full embedding described at the end of Section 4.1.] Without loss of generality, we may suppose that $e$ is the inclusion map, i.e. we will regard the point-set $P$ of $\mathbb{E}_{3}$ as a subset of the point-set of $D H(5,4)$. By the following lemma, we can without any ambiguity talk about distances between points of $P$.

Lemma $4.1 e$ is an isometric embedding.

Proof. Let $x \in P$. The number of points of $D H(5,4)$ at distance at most 2 from $x$ is equal to $\left|\Gamma_{0}(x)\right|+\left|\Gamma_{1}(x)\right|+\left|\Gamma_{2}(x)\right|=1+42+336=379$. Since $379<|P|=567$, there exists a point $y \in P$ which has distance 3 from $x$ in 
the dual polar space $D H(5,4)$. Recall that by Huang [17, Corollary 3.3], a full embedding between two dense near hexagons $\mathcal{S}_{1}$ and $\mathcal{S}_{2}$ is isometric if and only if there exist opposite points $x_{1}$ and $x_{2}$ in $\mathcal{S}_{1}$ which are mapped to opposite points of $\mathcal{S}_{2}$. Applying this here to the points $x$ and $y$, we see that the embedding $e$ is isometric.

If $Q$ is a quad of $\mathbb{E}_{3}$, then we denote by $\bar{Q}$ the unique quad of $D H(5,4)$ containing all points of $Q$. If $x_{1}$ and $x_{2}$ are two points of $Q$ at distance 2 from each other, then $\bar{Q}$ is the unique quad of $D H(5,4)$ containing $x_{1}$ and $x_{2}$. Recall that every quad of $\mathbb{E}_{3}$ is isomorphic to either $W(2)$ or $Q^{-}(5,2)$. Since every local space of $\mathbb{E}_{3}$ is isomorphic to $\overline{W(2)}$, each point is contained in precisely $15 W(2)$-quads and $6 Q^{-}(5,2)$-quads.

Lemma 4.2 (1) The map $Q \mapsto \bar{Q}$ defines a bijection between the set of quads of $\mathbb{E}_{3}$ and the set of quads of $D H(5,4)$.

(2) $P$ is a locally subquadrangular hyperplane of $D H(5,4)$.

Proof. (1) If $Q_{1}$ and $Q_{2}$ are two distinct quads of $\mathbb{E}_{3}$, then for every point $x_{1} \in Q_{1} \backslash Q_{2}$, there exists a point $x_{2} \in Q_{2}$ opposite to it. Hence, the map $Q \mapsto \bar{Q}$ is injective. The total number of $W(2)$-quads of $\mathbb{E}_{3}$ is equal to $\frac{|P| \cdot 15}{15}=567$ and the total number of $Q^{-}(5,2)$-quads of $\mathbb{E}_{3}$ is equal to $\frac{|P| \cdot 6}{27}=126$. Since $567+126=693$ is the total number of quads of $D H(5,4)$, the map $Q \mapsto \bar{Q}$ is necessarily bijective.

(2) Let $L$ be an arbitrary line of $D H(5,4)$ not contained in $P$ and let $\bar{Q}$ be an arbitrary quad of $D H(5,4)$ through $L$ corresponding to the quad $Q=\bar{Q} \cap P$ of $\mathbb{E}_{3}$. Since the $W(2)$-subquadrangle $Q$ of $\bar{Q}$ is a hyperplane of $\bar{Q},|L \cap P|=1$. Hence, $P$ is a hyperplane of $D H(5,4)$. Since every quad of $D H(5,4)$ intersects $P$ in either the whole quad or a $W(2)$-subquadrangle, $P$ is a locally subquadrangular hyperplane.

Definition. Let $(y, Q)$ be a point-quad pair of $\mathbb{E}_{3}$ such that $\mathrm{d}(y, Q)=2$. Then $\widetilde{Q} \cong W(2)$ and $\Gamma_{2}(y) \cap Q$ is an ovoid $\left\{y_{1}, y_{2}, \ldots, y_{5}\right\}$ of $\widetilde{Q}$. For every $i \in$ $\{1, \ldots, 5\}$, let $O_{i}$ be the unique ovoid of the $W(2)$-quad $\left\langle y, y_{i}\right\rangle_{\mathbb{E}_{3}}$ containing the points $y$ and $y_{i}$. Then $O_{1} \cup O_{2} \cup \cdots \cup O_{5}$ is called a nice set of points of $\mathbb{E}_{3}$. Every nice set of points of $\mathbb{E}_{3}$ contains precisely 21 elements. By the following lemma, a nice set consists of points at mutual distance 2 from each other. 
Lemma 4.3 For every point $x$ of $D H(5,4)$ not contained in $P, x^{\perp} \cap P$ is a nice set of points of $\mathbb{E}_{3}$. Conversely, for every nice set $N$ of points of $\mathbb{E}_{3}$, there exists a unique point $x$ of $D H(5,4)$ not contained in $P$ such that $N=x^{\perp} \cap P$.

Proof. (1) On each of the 21 lines of $D H(5,4)$ through $x$, there exists a unique point of $P$. Hence, $x^{\perp} \cap P$ is a set of 21 points at mutual distance 2. Let $\bar{Q}$ be an arbitrary quad through $x$ corresponding to a $W(2)$-quad $Q$ of $\mathbb{E}_{3}$ and let $y$ be an arbitrary point of $x^{\perp} \cap P$ such that the line $x y$ is not contained in $\bar{Q}$. Then $\mathrm{d}(y, Q)=2$ (since $\left.\Gamma_{1}(y) \cap \bar{Q}=\{x\}\right)$ and $\{y\} \cup\left(\Gamma_{2}(y) \cap Q\right) \subseteq x^{\perp} \cap P$. Put $\Gamma_{2}(y) \cap Q=\left\{y_{1}, \ldots, y_{5}\right\}$ and let $O_{i}$, $i \in\{1, \ldots, 5\}$, denote the unique ovoid of the quad $\left\langle y, y_{i}\right\rangle_{\mathbb{E}_{3}}$ containing $y$ and $y_{i}$. For every $i \in\{1, \ldots, 5\}, x^{\perp} \cap\left\langle y, y_{i}\right\rangle_{\mathbb{E}_{3}}$ is an ovoid of $\left\langle y, y_{i}\right\rangle_{\mathbb{E}_{3}}$ containing $y$ and $y_{i}$. (Notice that $x \in\left\langle y, y_{i}\right\rangle_{D H(5,4)}$.) Hence, $O_{1} \cup O_{2} \cdots \cup O_{5} \subseteq x^{\perp} \cap P$. Since both sets contain precisely 21 points, $O_{1} \cup \cdots \cup O_{5}=x^{\perp} \cap P$. Hence, $x^{\perp} \cap P$ is a nice set of points of $\mathbb{E}_{3}$.

(2) Let $(y, Q)$ be a point-quad pair of $\mathbb{E}_{3}$ such that $\mathrm{d}(y, Q)=2$. Put $\Gamma_{2}(y) \cap Q=\left\{y_{1}, \ldots, y_{5}\right\}$ and let $O_{i}, i \in\{1, \ldots, 5\}$, denote the unique ovoid of $\left\langle y, y_{i}\right\rangle_{\mathbb{E}_{3}}$ containing $y$ and $y_{i}$. Since $\mathrm{d}(y, Q)=2, y \notin \bar{Q}$. Let $x$ denote the unique point of $\bar{Q}$ collinear with $y$. Then the line $x y$ is not contained in $\bar{Q}$ and $\Gamma_{2}(y) \cap Q=\Gamma_{1}(x) \cap Q$. Repeating the argument exposed in part (1) of the proof, we find that $O_{1} \cup \cdots \cup O_{5}=x^{\perp} \cap P$. Conversely, if $z$ is a point of $D H(5,4)$ not contained in $P$ such that $z^{\perp} \cap P=O_{1} \cup \cdots \cup O_{5}$, then since $\left\{y_{1}, \ldots, y_{5}\right\} \subseteq \Gamma_{1}(z)$, we have $z \in \bar{Q}$. Since also $y \in \Gamma_{1}(z), z$ is necessarily equal to $x$.

Now, suppose $N$ is a nice set of points of $\mathbb{E}_{3}$ and let $y$ be an arbitrary point of $N$. Let $x$ be the unique point of $D H(5,4)$ not contained in $P$ such that $N=x^{\perp} \cap P$ and let $x^{\prime}$ denote the unique third point on the line $x y$. For every $z \in N \backslash\{y\}, \mathrm{d}(y, z)=2$ and $\langle y, z\rangle_{\mathbb{E}_{3}} \cap N=\langle y, z\rangle_{\mathbb{E}_{3}} \cap x^{\perp}$ is an ovoid of the $W(2)$-quad $\langle y, z\rangle_{\mathbb{E}_{3}}$ of $\mathbb{E}_{3}$. (Notice that $x \in\langle y, z\rangle_{D H(5,4)}$.) Hence, there exist $W(2)$-quads $Q_{1}, \ldots, Q_{5}$ of $\mathbb{E}_{3}$ through $y$ and for every $i \in\{1, \ldots, 5\}$ an ovoid $O_{i}$ of $Q_{i}$ through $y$ such that $N=O_{1} \cup \cdots \cup O_{5}$. For every $i \in\{1, \ldots, 5\}$, there exists a unique ovoid $O_{i}^{\prime}$ of $Q_{i}$ through $y$ distinct from $O_{i}$. Clearly, $x^{\prime \perp} \cap Q_{i}=O_{i}^{\prime}$ (notice $x, x^{\prime} \in \overline{Q_{i}}$ ) and $N^{\prime}:=O_{1}^{\prime} \cup \cdots \cup O_{5}^{\prime}$ is the nice set of points of $\mathbb{E}_{3}$ corresponding to $x^{\prime}$. The set $N^{\prime}$ is completely determined by $N$ and $y$. We say that $N^{\prime}$ is conjugate to $N$ with respect to $y$.

In Lemma 4.3, we proved that the points of $D H(5,4)$ not contained in $P$ are in bijective correspondence with the nice set of points of $\mathbb{E}_{3}$. In terms of 
this bijective correspondence, the lines of $D H(5,4)$ not contained in $P$ can be easily expressed. They correspond to the sets $\left\{y, N, N^{\prime}\right\}$, where $N$ is a nice set of points of $\mathbb{E}_{3}, y \in N$ and $N^{\prime}$ is conjugate to $N$ with respect to $y$.

We are now ready to give a proof of Theorem 1.3. Suppose $e_{1}$ and $e_{2}$ are two full embeddings of $\mathbb{E}_{3}$ into the dual polar space $D H(5,4)$. For every point $x$ of $\mathbb{E}_{3}$, we define $\theta\left[e_{1}(x)\right]:=e_{2}(x)$. For every point $y$ of $D H(5,4)$ not contained in $e_{1}(P)$, let $\theta(y)$ be the unique point $z$ of $D H(5,4)$ not contained in $e_{2}(P)$ such that $e_{1}^{-1}\left(y^{\perp} \cap e_{1}(P)\right)=e_{2}^{-1}\left(z^{\perp} \cap e_{2}(P)\right)$. By the previous paragraph, $\theta$ is an automorphism of $D H(5,4)$. Clearly, $\theta \circ e_{1}=e_{2}$. Obviously, $\theta$ is the unique automorphism of $D H(5,4)$ satisfying this latter property.

\section{Proof of Theorem 1.2}

\subsection{The uniqueness of the locally subquadrangular hy- perplane of $D H(5,4)$}

The aim of this subsection is to give a computer-free proof of the following proposition.

Proposition 5.1 Up to isomorphism, there exists a unique locally subquadrangular hyperplane of $\mathrm{DH}(5,4)$.

Proposition 5.1 is due to Pasini and Shpectorov [24, Theorem 1.3]. However, their proof relies on Pasechnik's computer classification of the hyperovals of $H(5,4)$. We will now give an alternative computer-free proof.

Lemma 5.2 Let $H$ be a locally subquadrangular hyperplane of $D H(5,4)$. Then there exists no point $x \in H$ such that $x^{\perp} \subseteq H$.

Proof. We will prove the following:

(*) If $x_{1}$ is a point of $H$ satisfying $x_{1}^{\perp} \subseteq H$, then also every point $x_{2} \in$ $\Gamma_{1}\left(x_{1}\right)$ satisfies $x_{2}^{\perp} \subseteq H$.

Let $x_{3}$ be an arbitrary point of $x_{2}^{\perp}$. Then there exists a quad $Q$ containing the points $x_{1}, x_{2}$ and $x_{3}$. Since $x_{1}^{\perp} \cap Q \subseteq H$, the quad $Q$ is contained in $H$. Hence, $x_{3} \in H$. This indeed implies that $x_{2}^{\perp} \subseteq H$. 
Now, suppose there exists a point $x \in H$ such that $x^{\perp} \subseteq H$. By successive application of $(*)$, we find that every point of $D H(5,4)$ is contained in $H$ which is clearly absurd.

Lemma 5.3 If $x_{1}$ and $x_{2}$ are two points of a locally subquadrangular hyperplane $H$ of $D H(5,4)$, then the distance between $x_{1}$ and $x_{2}$ in the geometry $\widetilde{H}$ is equal to the distance between $x_{1}$ and $x_{2}$ in the dual polar space $D H(5,4)$. As a consequence, $\widetilde{H}$ is a dense near hexagon with three points on each line.

Proof. In order to prove the first claim, it suffices to show that for any two points $x_{1}$ and $x_{2}$ of $H$ at distance at least 2 from each other, there exists a line $L \subseteq H$ through $x_{2}$ containing a point at distance $\mathrm{d}\left(x_{1}, x_{2}\right)-1$ from $x_{1}$. Consider a quad $Q$ through $x_{2}$ contained in $\left\langle x_{1}, x_{2}\right\rangle$ and let $L$ be a line through $x_{2}$ contained in $Q \cap H$. Then $L$ indeed contains a point at distance $\mathrm{d}\left(x_{1}, x_{2}\right)-1$ from $x_{1}$.

Since distances in $\widetilde{H}$ are the same as distances in the near polygon $D H(5,4), \widetilde{H}$ itself is also a near polygon. One can readily verify that the maximal distance between two points of $H$ is equal to 3 . So, $\widetilde{H}$ is a near hexagon with three points on each line. If $x_{1}$ and $x_{2}$ are two points of $\widetilde{H}$ at distance 2 from each other, then $\left\langle x_{1}, x_{2}\right\rangle \cap H$ is either $\left\langle x_{1}, x_{2}\right\rangle$ or a $W(2)$ subquadrangle of $\left\langle x_{1}, x_{2}\right\rangle$. Hence, $\left|\Gamma_{1}\left(x_{1}\right) \cap \Gamma_{1}\left(x_{2}\right) \cap H\right| \geq 3$ and $\widetilde{H}$ is a dense near hexagon.

Lemma 5.4 Let $H$ be a locally subquadrangular hyperplane of $D H(5,4)$. Then for every point $x \in H, \Lambda_{H}(x)$ is the complement of a hyperoval of $\operatorname{Res}(x) \cong \mathrm{PG}(2,4)$. As a corollary, every local space of $\widetilde{H}$ is isomorphic to $\overline{W(2)}$.

Proof. The complement of $\Lambda_{H}(x)$ (in $\operatorname{Res}(x)$ ) is nonempty by Lemma 5.2 and intersects every line of $\operatorname{Res}(x)$ in either 0 or 2 points (every quad through $x$ is either contained in $H$ or intersects $H$ in a $W(2)$-subquadrangle). Hence, $\Lambda_{H}(x)$ is the complement of a hyperoval of $\operatorname{Res}(x)$. The linear space induced by $\operatorname{Res}(x)$ on $\Lambda_{H}(x)$ is easily seen to be isomorphic to $\overline{W(2)}$.

Corollary 5.5 For every locally subquadrangular hyperplane $H$ of $D H(5,4)$, $\widetilde{H} \cong \mathbb{E}_{3}$.

Proof. This follows from the fact that $\mathbb{E}_{3}$ is, up to isomorphism, the unique dense near hexagon which satisfies the following properties: (1) every line is 
incident with precisely 3 points; (2) every local space is isomorphic to $\overline{W(2)}$.

We are now able to give a computer-free proof of Proposition 5.1. Suppose $H_{1}$ and $H_{2}$ are two locally subquadrangular hyperplanes of $D H(5,4)$. By corollary $5.5, \widetilde{H_{1}} \cong \widetilde{H_{2}} \cong \mathbb{E}_{3}$. Theorem 1.3 then implies that there exists an automorphism of $\mathrm{DH}(5,4)$ mapping $\mathrm{H}_{1}$ to $\mathrm{H}_{2}$.

\subsection{The uniqueness of the hyperoval of size 126 of $H(5,4)$}

For every set $X$ of points of $H(5,4)$, let $H_{X}$ denote the set of all singular planes of $H(5,4)$ which contain at least one element of $X$. We can regard $H_{X}$ as a set of points of $D H(5,4)$.

The following proposition is essentially contained in Pasini and Shpectorov [24]. However, the proof of part (1) relies on Pasechnik's computer classification of the hyperovals of $H(5,4)$ (see [24, Proposition 1.1]). So, an alternative proof is necessary for our purposes.

Lemma 5.6 (1) If $X$ is a hyperoval of size 126 of $H(5,4)$, then $H_{X}$ is a locally subquadrangular hyperplane of $\mathrm{DH}(5,4)$.

(2) If $H$ is a locally subquadrangular hyperplane of $D H(5,4)$, then there exists a unique hyperoval $X$ of size 126 of $H(5,4)$ such that $H=H_{X}$.

Proof. (1) Let $L$ be a line of $H(5,4)$. If $L$ contains 2 points of $X$, then each of the 3 singular planes of $H(5,4)$ through $L$ belongs to $H_{X}$. If $L$ is disjoint from $X$, then by Lemma 3.3, precisely one singular plane through $L$ belongs to $H_{X}$. Hence, $H_{X}$ is a hyperplane of $D H(5,4)$. In order to show that $H_{X}$ is a locally subquadrangular hyperplane, we need to prove that any two points $\alpha$ and $\beta$ of $H_{X}$ at distance 2 from each other have either 3 or 5 common neighbours which are contained in $H_{X}$. We will regard $\alpha$ and $\beta$ as singular planes of $H(5,4)$ and distinguish two cases:

(i) $\alpha \cap \beta \subseteq X$. Then each common neighbour of $\alpha$ and $\beta$ in $D H(5,4)$ belongs to $H_{X}$. So, $\alpha$ and $\beta$ have 5 common neighbours which are contained in $H_{X}$.

(ii) $\alpha \cap \beta \nsubseteq X$. Put $\alpha \cap \beta=\{x\}$. Let $L_{1}, L_{2}, L_{3}$ be the three lines of $\alpha$ through $x$ which intersect $X$ in 2 points. For every $i \in\{1,2,3\}$, let $M_{i}$ denote the unique line of $\beta$ through $x$ such that $\left\langle L_{i}, M_{i}\right\rangle$ is a singular 
plane of $H(5,4)$. If $\gamma$ is a common neighbour of $\alpha$ and $\beta$ belonging to $H_{X}$, then $\alpha \cap \gamma \in\left\{L_{1}, L_{2}, L_{3}\right\}$. (Recall that every singular line of $H(5,4)$ disjoint from $X$ is contained in a unique singular plane meeting $X$.) Hence, the neighbours of $\alpha$ and $\beta$ which are contained in $H_{X}$ are precisely the singular planes $\left\langle L_{i}, M_{i}\right\rangle, i \in\{1,2,3\}$.

(2) Let $H$ be a locally subquadrangular hyperplane of $D H(5,4)$, let $\mathcal{Q}$ be the set of quads of $D H(5,4)$ contained in $H$ and let $X$ denote the set of points of $H(5,4)$ corresponding to the quads of $\mathcal{Q}$. If $L$ is a line of $D H(5,4)$ contained in $H$, then by Lemma 5.4, precisely 2 quads through $L$ are contained in $H$. If $L$ is a line of $D H(5,4)$ not contained in $H$, then no quad through $L$ is contained in $H$. Hence, $X$ is a hyperoval of $H(5,4)$. By Lemma 5.4 , precisely 6 quads through a given point of $H$ are contained in $H$. Hence, $H=H_{X}$ and $|X|=\frac{v \cdot 6}{27}=126$ where $v=567$ is the total number of points of $\widetilde{H} \cong \mathbb{E}_{3}$. Conversely, if $Y$ is a hyperoval of size 126 of $H(5,4)$ such that $H=H_{Y}$, then every quad of $D H(5,4)$ corresponding to a point of $Y$ is contained in $H$. This implies that $Y=X$.

Theorem 1.2 is now a corollary of the following facts:

- the bijective correspondence between the hyperovals of size 126 of $H(5,4)$ and the locally subquadrangular hyperplanes of $D H(5,4)$ as explained in Lemma 5.6;

- the uniqueness, up to isomorphism, of the locally subquadrangular hyperplane of $D H(5,4)$ (Proposition 5.1);

- the fact that every automorphism of $D H(5,4)$ is induced by an automorphism of $H(5,4)$ (and vice versa).

\section{References}

[1] M. Aschbacher. Flag structures on Tits geometries. Geom. Dedicata 14 (1983), 21-32.

[2] R. J. Blok and A. E. Brouwer. The geometry far from a residue. Groups and geometries (Siena, 1996), 29-38, Trends Math., Birkhäuser, Basel, 1998.

[3] A. Blokhuis and A. E. Brouwer. Uniqueness of a Zara graph on 126 points and non-existence of a completely regular two-graph on 288 points. EUT-Rep., Eindhoven 84-WSK-03 (1984), 6-19. 
[4] A. E. Brouwer, A. M. Cohen, J. I. Hall and H. A. Wilbrink. Near polygons and Fischer spaces. Geom. Dedicata 49 (1994), 349-368.

[5] A. E. Brouwer and H. A. Wilbrink. The structure of near polygons with quads. Geom. Dedicata 14 (1983), 145-176.

[6] F. Buekenhout and X. Hubaut. Locally polar spaces and related rank 3 groups. J. Algebra 45 (1977), 391-434.

[7] P. J. Cameron. Dual polar spaces. Geom. Dedicata 12 (1982), 75-85.

[8] P. J. Cameron, D. R. Hughes and A. Pasini. Extended generalized quadrangles. Geom. Dedicata 35 (1990), 193-228.

[9] A. M. Cohen and E. E. Shult. Affine polar spaces. Geom. Dedicata 35 (1990), 43-76.

[10] B. De Bruyn. On the uniqueness of near polygons with three points on every line. European J. Combin. 23 (2002), 523-528.

[11] B. De Bruyn. Near Polygons. Birkhäuser, Basel, 2006.

[12] B. De Bruyn. Isometric full embeddings of $D W(2 n-1, q)$ into $D H(2 n-$ 1, $\left.q^{2}\right)$. Finite Fields Appl. 14 (2008), 188-200.

[13] B. De Bruyn. Two new classes of hyperplanes of the dual polar space $D H(2 n-1,4)$ not arising from the Grassmann-embedding. Linear Algebra Appl. 429 (2008), 2030-2045.

[14] A. Del Fra, D. Ghinelli and S. E. Payne. $(0, n)$-sets in a generalized quadrangle. Combinatorics '90 (Gaeta, 1990). Ann. Discrete Math. 52 (1992), 139-157.

[15] J. W. P. Hirschfeld. Finite projective spaces of three dimensions. Oxford Mathematical Monographs. Oxford Science Publications. The Clarendon Press, Oxford University Press, New York, 1985.

[16] J. W. P. Hirschfeld. Projective geometries over finite fields. Second edition. Oxford Mathematical Monographs. The Clarendon Press, Oxford University Press, New York, 1998. 
[17] W-l. Huang. Adjacency preserving mappings between point-line geometries. Innov. Incidence Geom. 3 (2006), 25-32.

[18] A. A. Makhnev. Extensions of GQ(4,2), the description of hyperovals. Discrete Math. Appl. 7 (1997), 419-435.

[19] A. A. Makhnev. Locally GQ(3,5)-graphs and geometries with short lines. Discrete Math. Appl. 8 (1998), 275-290

[20] D. Pasechnik. Geometric characterization of the sporadic groups $\mathrm{Fi}_{22}$, $\mathrm{Fi}_{23}$, and $\mathrm{Fi}_{24}$. J. Combin. Theory Ser. A 68 (1994), 100-114.

[21] D. Pasechnik. Extending polar spaces of rank at least 3. J. Combin. Theory Ser. A 72 (1995), 232-242.

[22] D. Pasechnik. The triangular extensions of a generalized quadrangle of order (3,3). Bull. Belg. Math. Soc. Simon Stevin 2 (1995), 509-518.

[23] D. Pasechnik. The extensions of the generalized quadrangle of order (3,9). European J. Combin. 17 (1996), 751-755.

[24] A. Pasini and S. Shpectorov. Uniform hyperplanes of finite dual polar spaces of rank 3. J. Combin. Theory Ser. A 94 (2001), 276-288.

[25] S. E. Payne and J. A. Thas. Finite Generalized Quadrangles. Research Notes in Mathematics 110. Pitman, Boston, 1984.

[26] E. E. Shult. On Veldkamp lines. Bull. Belg. Math. Soc. Simon Stevin 4 (1997), 299-316.

[27] E. E. Shult and A. Yanushka. Near $n$-gons and line systems. Geom. Dedicata 9 (1980), 1-72.

[28] J. Tits. Buildings of Spherical Type and Finite BN-pairs. Lecture Notes in Mathematics 386. Springer, Berlin, 1974.

[29] F. Zara. Graphes liés aux espaces polaires. European J. Combin. 5 (1984), 255-290. 